\title{
Index of names and works
}

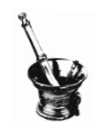

page numbers in italics refer to illustrations

Adams, Thomas

Mystical Bedlam 36-8, 45, 77, 283

Alberti, Salomon 38-9, 39

'De scorbuto' 249

'Schorbuti historia' 232

Aldersey, Dorothy, née Hudson

$$
\text { I40n., I43n. }
$$

Amatus Lusitanus

Curationum medicinalium ... duae $227,235,283$

Curationum medicinalium ... quatuor 20, 32, 62, 90, II3, I95, 245, $246,247,283$

Curationum ... quinta et sexta 37

Archer (Mrs) (Case I52) 234-5

Aurifaber

'Succini historia' I35

Austin, Alice (Case 43) I20

Baker, Daniel i97n.

Baker, Katherine 70-I, (Case I27) I97

Baker, Margaret (Case I4I) 2 I9

Ballard, James 63-4, (Case II2) I80-I

see also Ballard, James, of

Ombersley

Ballard, James, of Ombersley i8on.
Barnard, Lady Elizabeth, see Nash,

Elizabeth, née Hall

Barnard, Sir John i inn., Igon.

Barnes, Elizabeth, formerly

Rainsford $156 n$.

Barnes, Jane, née Smith $5_{56 n}$.

Barnes, Mary, of Talton (Case 55)

I30-I, (Case 62) I34

Barnes, Mary, of Tredington I5In.

Barnes, William, of Clifford

Chambers 6I, I5In, (Case 9o) ${ }^{5} 5^{6-7}$

Barnes, William, of Tredington 59, i3on., I49n., (Case 8I) I5I-2, I56n.

Barrough, Philip

Method of Physick, The 30, 3o, 288-97

Basse, Christiana, of Southam (Case 9) 87-8, 99n., 208n.

Basset (Captain) (Case 27) 99-Ior, (Case 34) ro9-io

Beale, William 246n.

Beaufou, Ann, Lady, née Aldersey I40n., (Case 73) I43-4, I45,

(Case 84) I53-4

Beaufou, Sir Thomas (Case 7o)

$$
\text { I40-I, I43n. }
$$




\section{Index of names and works}

Beaufou, Lady Ursula, née Hudson I40n.

Beaumont, Sir Francis 222n.

Béguin, Jean 42, 44 Tyrocinium chymicum 37, 229, 283

Benlow, Andrew i93n.

Bernard de Gordon 2 I 6

Lilium medicinae 37, 192, 203, 283

Bettes ('Goodwife') (Case 23) 95-7

Bettes (Mrs), of Ludlow (Case 5)

$$
83^{-}-5,95 \text {. }
$$

Betts, William 95n.

Bible 25

Job $237 n$.

Samuel 25, 237n., 239

Tobit 20on.

Wisdom 237n, 239

Bird, John 33-4, 67

Bishop ('the son of Mr') 62, (Case

59) $132-3$

see also Bishop, George; Bishop,

William

Bishop, Anthony I32n.

Bishop, George I32n.

Bishop, John i32n.

Bishop, William I32n.

Blencowe, Isabella, see Woodward, Isabella, née Blencowe

Boles (Mr) 25, 57

Boughton, Edward I39n., 20on.

Boughton, Elizabeth, née Catesby xvii, (Case 69) I39-40, 200 .

Boughton, Joyce (Case 24) 97, (Case 26) $98-9$

Bovey, John $247 n$.

Bovey, Margaret, née Harrison 62, (Case I6r) 247

Brackenburgh, Richard pl. I

Brent, Dixwell 62, (Case 94) I58-9

Broad, Frances, née Badger i64n.

Broad, William 6o, 88, (Case Ioo) I64-5

'Mr Broad's tutor' 88

'Browne' ('Romish priest') (Case 33) IO7-9
Browne, (Mrs) Bridget, see

Throckmorton, Bridget

Browne, Elizabeth, Lady 63, 70,

(Case Io6) I72-4, (Case I62)

248-50

Browne, George i72n.

Browne, Sir William i72n.

Bruele, Walter [Gualtherus] 44

Praxis medicinae 37, 80, 84, 86, I05, I52, I64, I66, I83, I92, 209, 2I $4,236,273-4,283$

Burman, John I4, I4

Burton, Robert Anatomy of Melancholy, The 37, 40-I, 4I, 45, I68, I83, 272, 283

Butler 63, (Case I2I) I9I-2 see also Butler, Robert

Butler, Robert (glover) I9In.

Butler, Robert (surgeon) IgIn.

Cardano, Girolamo 43 Ars curandi parva 37, 43, II6, 283

Castro, Rodrigo de

De universa mulierum medicina 37 , I I5, I77, 284

Catesby, Edward i39n.

Cecil, Edward, Viscount

Wimbledon 228

Chandler (Mrs) (Case 8) 87, (Case 28) IOI

Chandler, William 87n.

Charles I, King of England I2, I5, IOIn., I78n., 2I0, 28In.

Cicero (Marcus Tullius Cicero)

De re publica I04n., I57n., 255n., 28In.

Glark, Dorothy, Lady, née Hobson, formerly Hay (Case I3I) 20I-2, 208n.

Clark, Margaret, née Alderford 208n.

Clark, Sir Simon 20In., 202n., (Case I36) 208

Clavell, William 59, (Case 76) I46-7

Clayton, Dr Thomas 66-7, 2 I0 


\section{Index of names and works}

Clopton, Anne, née Clopton i8In.

Clopton, Elizabeth i8In.

Clopton, John i8In.

Clopton, William i8In.

Colemore, William 25

Collins, Alice, see Greene, Alice, née Collins

Collins, Francis I9, I95n.

Combe, Katherine, née Boughton I9, 62, I39, (Case I30) 200-I

Combe, Mary, see Wagstaffe, Mary, née Combe

Combe, William I4, I4, I9, I39

Compton, Lady Elizabeth, see Northampton, Elizabeth, Ist Countess

Compton, Lady Mary, see Northampton, Mary, née

Beaumont, 2nd Countess

Compton, Spencer, see Northampton, Spencer Compton, 2nd Earl

Compton, William, Baron, see Northampton, William Compton, ist Earl

Conway, Sir John 204n.

Cooke, James I8-2I, 42, 5I, 5I, 54, $56,5^{8}$

Mellificum chirurgiae I9, 42

Select Observations 20-I, 28-34, 42, $5^{6-9}, 63,65,66-72,68,69$, 85n., I I7n., I54n., I76n., I92n., 246n., 255n.

Cookes (Mrs) (Case I73) 269-70 see also Cookes, William; Cookes, Alice

Cookes, Alice 269n.

Cookes, William 26gn.

Cooper, Mary, née Martin (Case 46) I2I-2, I33

Cooper, Thomas I2In.

Cotta, John 59

Court, Grace, later Pargetter 6o-I, (Case I43) 220-2

Court, John, the elder $220 n$.
Court, John, the younger 220 .

Cox, Hester, née Sylvester, see Sylvester, Hester

Cox, Richard i95n.

Crato, Johannes

Consiliorum et epistolarum 32, 37-8, 44, 48-9, 6I, 79, 8I, 92-3, 95, 97, II0, I28, I35, I6I, I68, I74, I87, I89, I99, 202, 2 16, 284, 290

Croll, Oswald

Basilica chymica I6, 32, 64, 86, I54, I8I, I92, 272, 284

Cromwell, Oliver i24n.

da Monte, Giovanni Battista 40, $43-4$

Consultationum medicinalium 32, 64, 9I, I09, 284

Consultiones medicae 37, I91, 284

Davenport, Sidrick 22-5, 22, 23

Davies (Mrs) (Case 58) I32 see also Davies, Frances

Davies, Frances I32n.

Davies, John I32n.

de Castro, Rodrigo, see Castro, Rodrigo de

de Fonseca, Rodrigo, see Fonseca, Rodrigo de

de Gordon, Bernard, see Bernard de Gordon

Deacle (Nurse) 63, (Case I74) 270-2

Delabere, Margaret 40, (Case I I I) I78-9

Delabere, Richard i78n.

Dodens, Rembert pl. 3

Donne, John 38, 25on.

Devotions upon Emergent Occasions 37-40, 45, 240, 284

Dornkrell, Tobias

Dispensatorium novum 32, I89, I9I, I93, I94, 280, 285

Drake, Sir Francis I03n.

Drayton, Michael (Case 22) I9, $95^{-6}, 9^{6,25 \text { on., 25 }}$ I

Idea $25 \mathrm{I}$ 


\section{Index of names and works}

Du Chesne, Joseph 34, 42, 45, 205

De priscorum philosophorum 37, 242, 276, 285

Diateticon polyhistoricon 32, I06, 285, 295

Pharmacopoea dogmaticorum 2I, 40, 45, 48, I04, II9, I40, I57, I79, 200, 226, 229, 276, 280, 285

Dudley, Sir Robert 230 .

Dugard, Thomas i72n., 206n.,

Dugdale, William

Antiquities of Warwickshire 89n., 97n., I29, I29, I3On., I74n., I93n., $275^{\text {n. }}$

Dunus, Thaddaeus

De curandi ratione 37, 83, 285

Dyck, Osias I02, pl. 25

Dyson, Thomas 63-4, (Case I6) 9I

Easton, John I4, I4

Echt, Johann 38-9, 39

Elizabeth I, Queen of England 8on., I4In., 208n., 210, 238n.

Emes, Elizabeth, née Bellers 89n.

Emes, John (Case I3) 89

Emes, John, the elder $89 n$.

Eugalenus, Severinus

De scorbuto morbo liber 2I, 32, 34, $43,48,63-4$, I69-70, I73-4, I8I, 200, 232, 237, 248-9, 252, $255^{-6,275}, 278,285$

Falloppio, Gabriele

'De morbi Gallico' 97n.

Farnham, Thomas (Case 74) I45

Fawcet, Margaret, née Sellers $225 \mathrm{n}$.

Fawcet, (Revd) Thomas (Case I45) $225^{-6}$

Fernel, Jean 8I, 43, 8I, 95, I37-8, 202, 206-7, 217, 242

Consiliorum liber 37, I67, I83, 285

Therapeutices universalis 37, 77

Ferrers, Edward I63n.
Ferriman, Magdalen, née Smalwait $238 n$.

Ferriman, (Revd) Thomas, the elder 238

Ferriman, (Revd) Thomas, the younger $238 \mathrm{n}$.

Feschville, Sir John 2I8n.

Feschville, Lady Sarah, née Harington, see Harington, Sarah

Fetherston, Isabel, née Woodward, see Woodward, Isabel

Fetherston, John 232n.

Feyens, Jean

De flatibus 37, I01, I04, I96, 28I

Field, Richard 30, 30

Fiennes, Frances, née Cecil 59, 63, (Case I47) 228-3o, (Case I67) 258-6I, 270

Fiennes, James, 2nd Viscount Saye and Sele I9, 228n.

Fiennes, William, Ist Viscount Saye and Sele 228

Finch, Frances, née Jones (Case I48) 230

Finch, Thomas $230 n$.

Fletcher, John 9

Fonseca, Rodrigo de

Consultationes medicae 33, 46, I93, I97, 225-6, 273-4, 285

Foreest, Pieter van 42, 44, I22, I69-70, I73, I78, I85, I89-9I, I94, I99-20I, 230, 24I, 249-50, 276,280

Observationum ... de febribus 37, 44, I69, 206, 212, 245-6, 26 г, 285

Forman, Simon 25I-2n.

Fortescue, Jane, née Wylde ioin.

Fortescue, Martha, see Lewis, Martha

Fortescue, Sir Nicholas i $18 n .$, (Case I68) 26I-3

Fortescue, Lady Prudence, née

Wheatley 26In.

Fortescue, William (Case 29) IоI-3 


\section{Index of names and works}

Galen I5, 3I, 43, I00, I03, IIO, I76, I87, I89, 24I

Gardner, Elizabeth (Case 32) Io7

Garner (Mrs), see Gardner, Elizabeth

Garrick, David $5^{8}$

Gerard, John The Herball pl. 3-20

Gesner, Conrad Historiae animalium 239-40

Gibbs, Ann (Case I2) 89

Goodere, Sir Henry 250n., 25I

Goodman (Mrs), see Goodman, Sara

Goodman, Sara (Case 96) I6I

Goodman, Thomas i6In.

Goodyeare (Mrs) 226

Gordon, Bernard de, see Bernard de Gordon

Greene, Alice, née Collins i9, (Case I24) 195

Greene, Anne I9, (Case 65) I36-7

Greene, John 3

Greene, Lettice i36n.

Greene, Thomas i95n.

Greene, Thomas ('Lawyer Greene') 8-9, I9, i36n., I95n.

Greene, William I36n.

Greville, Sir Edward I6I

Greville, Sir Fulke, ist Baron Brooke 204n., 257n.

Gunn, Ann, see Izod, Ann

Hall, Dive (brother) I2

Hall, Elizabeth, see Nash, Elizabeth, née Hall

Hall, John

education I-6, 29-3I, 33, 42-4, 46

illness 6o, (Case I55) 239-4I

marriage 6-II, 7, 8,17

medical career $2,4,17,6-27,3 \mathrm{I}$, $42,47-9,50-65,51,52,53,55$,

$66-7 \mathrm{I}, 68,69,78$

reading and library $3-5,5$, II, I5-2I, 25-7, 28-49, 39, 4I, $5^{\mathrm{I}-4}, 62-4,7 \mathrm{I}-3$ will, death and burial I, 7, I8, $25-7,26,42,57,83 n$.

Hall, Susanna, née Shakespeare I-3, 6-I4, I4, I6-I9, I7, 2I, 25-6, 26, 31, 56-8, (Case i9) 93, II2, (Case I29) I98-200, 27I

Hall, William I, 3, I2, 31, 36, 45, 46

Hanbury, Anne (later Thornborough) (Case I I) 88, (Case 87) I55

Hanslap, Margaret, née Hill I68n.

Hanslap, Robert (Case I04) I68-7I, I74

Harington, Sir John 2 I8n.

Harington, Sarah (Case I4I) 2I8-I9

Harrison, William 247n.

Hart, Joan, née Shakespeare II2

Hart, Thomas II 2

Hartmann, Johann 44

Praxis chymiatrica 37, 280, 285

Harvey, Sir Francis 59, (Case I39) 2 I4-I6

Harvey, Lady Mary, née Murden

(Case 6o) I33, I74n., (Case 93) I58, I59, 2 I4n.

Harvey, Sir Stephen I33n., 2 I4n.

Harvey, Dr William 2 I6

Hathaway, Bartholomew 2

Hay, William 20 in.

Heath, John $92 n$.

Heath, Mary (Case I7) 62, 92-3

Henrietta Maria, Queen of England 93n., $218 n$.

Henry Frederick, Prince of Wales 206n.

Herbert (Mrs) (Case 20) 93-4

Hippocrates I5, 3I, 43, 5I, 64-5, 77, 77n., 204, 270

Hitchcox, Isaac 4,4

Hobson, Thomas 20 in.

Holland, Thomas 236n.

Holle, William 96

Holyoak, (Revd) Francis 6r, 245

Holyoak, (Revd) Thomas 6i, (Case

I59) $245^{-6}$ 


\section{Index of names and works}

Hopper, Cecily (Case I5I) 234

Hopper, John 234n.

Horsey, Dorothy 257n.

Horsey, Fulca, née Swift, see Swift, Fulca

Horsey, James 257n.

Horst, Gregor

Observationum medicinalium 32, 45, 46, 48-9, г25, г69, г70, I86, I9I, 203, 206-7, 222, 224, 255, 26o-3, 263n., 265, 280

Houllier, Jacques

De morbis internis 20, 32, 42-3, IO3, II3, I29, 285

Hudson ('a poor man') 20, (Case 9I) $\mathrm{I} 57$

Hugges, Peter (Case I6o) 246-7

Hunks, Lady Katherine, née Conway 99n., I6rn., (Case I34) 204-5

Hunks, Sir Thomas 99n., 204n.

Hunt, Henry gon.

Hunt, Mary, née Russell (Case I5) 90-I

Iremonger (Mrs) 20, (Case I05) I7 I-2

Izod, Ann, formerly Gunn I86n.

Izod, Henry (Case I I7) I86

Izod, Henry, the younger i86n.

Jackson, Anne (Case I49) 230-2

Jackson, (Revd) John 23 on.

James I, King of England 99n., I39, I49n., 204n.

Jelphes, Joseph 85 n.

Jenkinson, Anne, Lady, née Lee

(Case I20) I90-I, I94

Jenkinson, Sir Robert igon.

John of Gaddesden

Praxis medica, rosa anglica 32, 34, 90-I, I9I-2, 285

Jonson, Ben 250 .

Judkin, Joan, née Twigge (Case 39) I $6-$ - 7
Judkin, Robert i 6 n.

Judkin, Thomas 87n.

Kempson, Leonard (Case 31) I04-7, I48n.

Kempson, Letitia I3In.

Kempson, Margaret, née Sadler 6o, (Case 78) I48-9

Kempson, Thomas I3In.

Kenton (Mrs) (Case i Io) I77-8

Kimberley (Mrs) 59, (Case I69) 263-6

Kimberley, Gilbert 263n.

Kimberley, Justinian 263n.

Kimberley, Robert, the elder $263 n$.

Kimberley, Robert, the younger $263 n$.

Kimberley, William $263 n$.

Kington, Elizabeth, née Warde (Case 44) I20-I

Lane, Joan (Case I8) 92-3

Lane, John I3

Lane, Richard 92n.

Lange, Johann 38-9, 39

Lapworth, Dr Edward 243, 266 see also Lapworth, William

Lapworth, William 243n.

Laud, William, Archbishop of Canterbury 15

Layton, Margaret, née Clopton 63, (Case II3) I8I-3

Lee, Sir Robert igon.

Leicester, Robert Dudley, Ist Earl

I87

Lewis, Sir Edward 236n.

Lewis, Martha, née Fortescue (Case 4I) Ir8, (Case I53) 236, 26In.

Lewis, Nicholas i $18 n ., 236 n$.

Liébault, Jean

Thresor de remedes secrets i I, 32-3, I07, I28, I77, I78, 285

Livy (Titius Livius)

Historiam ab urbe condita $\mathbf{1} 45$.

Lorkin, Thomas 29 


\section{Index of names and works}

Luisini, Luigi

De morbo Gallico omnia 37, 97, 28o, 285

Lynes, Humphrey, the elder ig6n.

Lynes, Humphrey, the younger I96n.

Lynes, Joan, née Richardson (Case I26) I96-7

Malone, Edmond $5^{8}$

Manning, Elizabeth 9in.

Mansfeldt, Ernst von 99n.

Marcus Aurelius 5I

De vita sua 77

Markham, Mary i26n.

Markham, Thomas I26n.

Martini, Matthaeus

'De scorbuto' 38-9, 39, 79, I49, І70-3, I8I-5, I99, 248-9, 256, $25^{8}$

Montagnana, Pietro da 6I, I4I, I54

Morley, Sir John I7In.

Morrys, John 3

Morrys, Matthew 3

Morrys, Susanna 3

Murden, Mary (later Lady Harvey), see Harvey, Lady Mary, née

Murden

Murden, Mary, of Moreton Murrell (Case I07) I74, I75

Murden, Richard I33n., I74n., I75

'Mr Nash's serving maid' 20, (Case 6) $85-6$ see also Nash, Anthony

Nash, Anthony 85n., I03-4n.

Nash, Elizabeth, née Hall (later Elizabeth, Lady Barnard) 6, I9-2 I, 25, 93n., I03-4n., (Case 36) I I I-I4, II2, I9on.

Nash, Mary, née Bough ig, (Case 30) $103-4$

Nash, Thomas 25, 57, I03-4n., IIIn.

Nason, Elizabeth, née Rogers i38n.

Nason, John 4, 4, (Case 67) I38
Nidd, John 29

Norbury, John I48n.

Northampton, Elizabeth Compton, née Spencer, ist Countess I9, (Case i) 77-8o, 82, 86, 88n., (Case 95) I59-6r, I67, pl. 2

Northampton, Mary, née Beaumont, 2nd Countess I9, 6o, 62-3, 208n., 2 Io, (Case I44) $222-5$

Northampton, William Compton, Ist Earl I9, 77n, (Case 2) 80-2, 82, 83n., 86n. 93n., 95n., (Case 64) I35-6, (Case 68) I38-9, (Case 79) I49, (Case 83) I52-3, 208, 2 Io, 246n., pl. 2

Northampton, Spencer Compton, 2nd Earl 66, (Case I37) 208-12, 210, 222n., 246n., (Case i78) $28 \mathrm{I}-2$

Occo, Albert

Pharmacopoeia Augustana 37, 45, I4I

Pakington, Dorothy, née Smith I4In., I42

Pakington, Sir John 6I, (Case 7I) I4I-2, (Case 85) I54, pl. 2

Palmer, Jane, née West (Case 54) I3O

Palmer, John izon.

Paracelsus II, I5, I44, I94, 202, 269

Pargetter, Christopher 220 .

Parker (Mr) (Case 52) I28-9 see also Parker, Henry

Parker, Henry i28n.

Peers, Clare, née Benlow 7o, (Case I23) I93-4

Peers, Philip i93n.

Peers, Thomas i93n.

Penell (Mr), see Pennell, Edward

Pennell, Edward (Case 97) I6I-2

Pennell, Margaret, née Greville i6 In. 


\section{Index of names and works}

Penot, Bernard

De denario medico 32, 42, 44, 88, I5I, 286

Pharmacopeia Londinensis (College of Physicians) I6, 32, 34, 45, 87, 9I, I4I, 2I7, 237-8, 268

Platter, Felix 20-I, 44-5, 6I

Observationum ... libri sex I84, 273

Observationum ... libri tres 37, 45, 48, 6I, IIo, II4-I6, I20, I23-5, I27-8, I30-I, I74, I77, 2I4-I5, 22I , 224, 230-2, 274, 286, 295

Praxeos ... de doloribus 32, 81, 83, 94-5, I34, I85-6, 286

Praxeos ... de functionem laesionibus 20, 32, II3, II5-I6, II9, I23, 286

Praxeos ... de vitiis 32, 37, 62, I 77-I8, I59, 286

Potier, Pierre 42, 44

Insignium curationum 37, 139, 278, 286

Pharmacopoea spagirica 37, 45, I78, 286

Potter, Lydia, née Trapp, see Trapp, Lydia

Potter, William i83n.

Powell (Mr) (Case 7) 86

Publilius Syrus

Sentences I65n.

Puckering, Elizabeth, Lady, née Morley 20, (Case I05) I7I-2, I95

Puckering, Sir John I72, 206n.

Puckering, Sir Thomas i7in., (Case I35) 206-8, (Case I56) 242-3

Quiney, George I9, (Case 38) II5-I6

Quiney, Judith, née Shakespeare I9, II5n.

Quiney, Thomas II5n.

Rainsford, Anne, Lady, née Goodere i9, 95n., 96, I49n., I5o, (Case I63) 250-I, $25^{I}$
Rainsford, Lady Elinor, née Boswell I9, (Case I42) 219-20

Rainsford, Elizabeth, see Barnes, Elizabeth, formerly Rainsford

Rainsford, Sir Henry, the elder I9, 6o, (Case 80) I49-5I, I5o, I56n., 250n., 25I

Rainsford, Sir Henry, the younger 2Ign.

Rainsford, Henry 2ign.

Rainsford, Hercules I49n.

Randolph, Dorothy, née Izod i86n.

Randolph, Edward I86n.

Randolph, Elizabeth (Case 6I) I33, I63n.

Randolph, Elizabeth, née Ferrers 6o, 97n., (Case 99) I63-4

Randolph, Elizabeth, the younger I33n.

Randolph, Ferrers (Case 25) 97-8, i33n., i63n., I86n.

Randolph, Thomas I33n., I63n.

Ranzau, Henrik

De conservanda 37, 79, I53, 286

Rawlins, Edward (Case 53) I3o

Reyland, Francisca (Case I4) 89-9o

Richardson (Mrs) (Case I22) I92-3, 200-I

see also Richardson, Elizabeth, née Burman

Richardson, Elizabeth, née Burman I92n.

Richardson, William ig2n.

Rivière, Jean Ribit de la 276-7

Roberts, Thomas ('Roberts Sartor') (Case 63) I35

Rogers, Frances (Case 98) i62-3

Rogers, (Revd) John (Case 72)

I 4 I-3

Rogers, Philip I62n.

Rondelet, Guillaume

Methodus curandorum 20, 33, 43, II3, 286

Ronsse, Baudouin

'De scorbuto' 38-9, 39, 20I, 276 


\section{Index of names and works}

Rous, Lady Esther, née Temple

(Case 75) I45-6, (Case 89) I56,

(Case 92) 157-8

Rous, John 2i6n.

Rous, Sir Thomas I45n.

Rueff, Jacob

De conceptu 37, 2I7, 234, 286

Ruland, Martin, the Elder I I, 25-7, $3^{8}, 4^{2}, 43^{-4}, 4^{8}$

Balnearium restauratum 98n., 286

Curationum empyricarum . . centuriae II, 2I, 25, 33, 38, 44, 48, 52, 70, 77n., 84-7, 97-9, Іог, Іо7, Іо9, II 4 , II7-20, I22, I24, I26-8, I34-7, I39-40, I42-4, I47-8, I52, I56-7, I6I, I64, I67, I74, I76, I79, I8I, I83-5, I87, I95-6, 20I, 205, 208, 2II-I2, 2I9, 222, $224-5,228-30,232 ., 235^{-6}$, $239,242^{-}-3,245,249,25^{8}, 26 \mathrm{I}$, 263, 266, 268-70, 275, 28I, 286, 289-90, 295

Ruland, Martin, the Younger

De morbo Ungarico 37, 45, 105 , I08-9, I43, 220-2, 23I, 286

Russell, Richard, of Flyford Flavell gon.

Russell, Thomas I9

Sadler, Isabel, née Smart (Case Ior) I65-6

Sadler, John I48n., i65n.,

Salisbury, Countess of, see Shrewsbury, Earldom of

Sandys, Edwin i i4n.,

Sandys, Penelope, Lady, née Bulkeley (Case 37) I I4-I5

Savage (Mrs) 226

Saxton, Christopher pl. 2

Saye and Sele, Lord, see Fiennes, James; Fiennes, William

Schenk von Graffenburg, Johannes $3^{8}$

Sellers, John 225n.

Seneca 272n.
Sennert, Daniel 42, 45

De febribus libri 37, 232, 286, 296

De scorbuto tractatus 2 I, 33, 34, $3^{8-9}, 39,44,48,63-4,79$, I49, I69-70, I73, I8I-3, I85, I99, 20I, 232, 237, 248-9, 252, 254, $256,258,260,264,276,286$

Medicina practica 37, 267, 287

Opera omnia 40

Shakespeare, Anne, née Hathaway I, 2, $136 \mathrm{n}$.

Shakespeare, William

life I, 2, 3, 6, 8-I I, I8-I9, 30, 58, 8in., 93n., 96, iıin., iı2, iा5n., i36n., I4I-2n., I95n., I98n. works All's Well That Ends Well 9; Comedy of Errors, The 9; King Lear io; Macbeth Io; Merry Wives of Windsor, The 9; Pericles Io-I I; Romeo and Fuliet I ; (with Fletcher) Two Noble Kinsmen, The 9; First Folio Io

Shaw, July 2, 8in.

'neighbour Sheffield, the sister of' (Case 88) I55

Sheffield, Elinor (Case 82) I52 see also 'neighbour Sheffield, the sister of'

Sheffield, John I52n.

Sheffield, Thomas i2on.

Sheldon, Brace I3In.

Sheldon, Edward I26n.

Sheldon, Elizabeth, née Markham (Case 50) I26-7

Sheldon, Elizabeth, of Weston (Case 5I) I27-9, I29, 21 2

Sheldon, Margaret, née Kempson (Case 57) $\mathrm{I} \mathrm{I}^{-2}-2$

Sheldon, William i27n., 212n.

Sheppard, Sara, née Hall 6

Sheppard, William 6

Sherley, John 54

Shrewsbury, Earldom of I9-20, I3In., I67n. see also Talbot 


\section{Index of names and works}

Simonds, George i24n.

Simonds, Isabel, née Penrice (Case 48) $124-5$

Simonds, Thomas i24n.

Smart, Peter i65n.

Smith (Mrs) (Case I28) I98

Smith, Anne, née Shaw 54, (Case 3) $8 \mathrm{I}$

Smith, Sir Charles (Viscount Carrington) I87n.

Smith, Lady Elizabeth 59, (Case i i8) I87-8

Smith, Francis 244n.

Smith, John 67-7o, (Case 66) I37

Smith, Margaret, see Sylvester, Margaret, née Smith

Solenander, Reiner I69

Consiliorum medicinalium 37, 80, I26, I33, I54, I69, 280, 287

Sprigge, Joshua 228n.

Stoker, Elizabeth 129 (Case I38) 2I $2-\mathrm{I} 3$

Stone, Nicholas 242n.

Stoughton, Anthony 266n.

Stoughton, Edith 59, 62-3 (Case I70) 266, (Case I75) 272-5

Sturley, Abraham 6, 203n.,

Sturley, Katherine 63, 64, (Case I33) 203-4

Swift, Sir Francis 257n.

Swift, Fulca 59, (Case I66) 257-8

Sylvester, Hester 62, (Case I25) I95, $244 \mathrm{n}$.

Sylvester, Margaret, née Smith I95n., (Case I58) 244-5

Sylvester, Paul i95n., 244n.

Symcotts, John

'Observations I636' 40

Symons, John (Case 45) I2 I

Talbot, George 62, (Case 56) I3I

Talbot, Gertrude, see Wintour, Gertrude

Talbot, Sir John I67n.
Talbot, Mary 62, (Case I03) I67-8, I94

Taranta, Valesco de

Epitome operis 20, 33, II I, 287

Temple, Lady $2 \mathrm{I}-2$

Temple, Sir Thomas, ist Baronet

Stowe 2I-2

Thornborough, Dr John, Bishop of

Worcester I3-I4, I4, 2I, 59, 62,

63, 70, (Case I64) 25I-5, 253,

pl. 2

Thornborough, John 88n.

Throckmorton, Bridget, née Browne (Case I02) I66-7, I72n., 248n.

Throckmorton, Clement I66n.

Throckmorton, George i72n.

Throckmorton, Lady Mary (Case 42) II 8-I9

Throckmorton, Sir Robert i i8n.

Torrella, Gaspar

'De dolore in pudendagra' $280 n$.

Trapp, (Revd) John 6o, I69n., I83-4, (Case I77) 277-8I, 279

Trapp, Lydia 6i, (Case I I4) I83-4, 279

Trapp, Mary, née Gibbard 277n.

Treasurie of Poor Men, The (anon.) 5

Tyler, Richard i9, (Case I57) 244

Tyler, Susanna, née Woodward $244 \mathrm{n}$.

Tyrrell, Lady Elizabeth 2I-2

Underhill, Lady Catherine, née Uvedale (Case I I5) I84-5, I85n.,

Underhill, Sir Edward I84n., (Case II6) I85-6

Underhill, Elizabeth, née Daston $202 n$.

Underhill, Elizabeth, formerly Savage 255n., 275n.

Underhill, George (Case I08) I74-6, (Case iog) I76-7, 202n.

Underhill, Sir Hercules 255n.

Underhill, Sir John 275n. 


\section{Index of names and works}

Underhill, Simon 63, (Case I65) $255^{-7}$

Underhill, Thomas I74n., (Case I32) 202-3, 275n.

Underhill, Thomas, the younger (Case I76) 275-7

Uvedale, Sir William i85n.

Valesco, see Taranta, Valesco de

Valleriola, François

Observationum medicinalium 25, 33, 43-4, 48, 62, 92, II5, I21, I56, I60, I64-5, I80-I, 2II, 222, 228, 237, 239-40, 262, 270, 272, 275,287

Vernon, John 236n.

Vernon, Susanna, née Holland 63, (Case I54) 236-9

Vettori, Benedetto

Exhortatio ad medicum recte 37, 95, I38, 287

Virgil (Publius Vergilius Maro) Aeneid I45n., I47n.

Vittori, Leonello

De aegritudinibus infantium 37, 62, I32, 287

Wagstaffe, Mrs Elizabeth (Case 47) I22-4, 2 I6n., (Case I72) 268-9

Wagstaffe, Mary, née Combe, later Rous (Case I40) 2I6-I8

Wagstaffe, Sir Thomas I22n, 216n., $268 n$.

Wagstaffe, Timothy i22n.

Walker, John 6I, (Case 35) i io

Walker, (Revd) Thomas 6i, i Ion.

Ward, Anne (Case I46) 226-7 see also Ward, Anne, née Cap

Ward, Anne, née Cap 226n.
Ward, Edward 226n.

West, John I25n., I3on.

West, Julian, née Hannes (Case 49) I25-6, I3on.

Wheler, Robert Bell 6

Wier, Johannes

'De scorbuto' 38-9, 39, 264

Wilkes, Edward 4

Willich, Jodocus

Urinarum probationes 37, 64, 204, 26I, 287

Wilmore, Joan, née Awry I47n.

Wilmore, Richard (Case 77) I47-8

Wilson (Mrs) (Case I7I) 266-7

'Wilson of Stratford' (Case 86) I54-5

Wilson, Mary (Case 2I) 94-5

Wilson, (Revd) Thomas 2, I3-I5, I4, 63, (Case 4) 83, I42n., 266n.

Wilson, William I4

Wincoll (Mrs) (Case Io) 42, 88

Winter (Mr) 62, (Case 40) II7

Winter, John I9, II7n., I88

Winter, Gertrude, née Talbot I67n.

Winter, Margaret, née Russell ı9, (Case I I9) I88-9

Winter, Robert I67n., I88

Winter, Thomas I88

Woodward, Isabel 59, 63, (Case I50) $232-4$

Woodward, Isabella, née Blencowe $232 n$.

Woodward, John 232n.

Wren, Christopher 242n.

Wright, Dr Nathaniel i67n.

Young, Edith, née Stoughton, see Stoughton, Edith

Young, Thomas 266n. 